\title{
Avaliação do nível de conhecimento de escolares do ensino médio sobre o câncer bucal
}

Assessment of the level of knowledge of high school students about mouth câncer

Evaluación del nivel de conocimiento de los estudiantes de secundaria sobre el cáncer de boca

Fernando Yamamoto CHIBA

Suzely Adas Saliba MOIMAZ

Cléa Adas Saliba GARBIN

Tânia Adas SALIBA

Marco Aurélio Borella RODRIGUES

Maria Lúcia Marçal Mazza SUNDEFELD

Departamento de Odontologia Preventiva e Restauradora, Universidade Estadual Paulista (UNESP), Faculdade de Odontologia de Araçatuba, 16015-050 Araçatuba - SP, Brasil

\section{Resumo}

O conhecimento da relação entre o câncer de boca e fatores relacionados ao estilo de vida, como o tabagismo, etilismo e a exposição solar são conhecidos na comunidade científica, entretanto, este conhecimento também deve ser disseminado nos diversos estratos da população. Objetivou-se avaliar o conhecimento de escolares do 3ํano do ensino médio de um município de médio porte do Estado de São Paulo sobre o câncer de boca. Método: Estudo transversal com 1012 escolares da rede pública de ensino de Araçatuba-SP, Brasil. Utilizou-se um questionário estruturado contendo questões sobre conhecimentos gerais; sintomatologia; fatores de risco; fatores de proteção; epidemiologia; prevenção e tratamento do câncer de boca. Os dados foram processados e analisados com auxílio do programa Epilnfo versão 7.2. A maioria dos alunos (93,9\%) havia ouvido falar sobre o câncer de boca, entretanto, pouco mais da metade $(60,1 \%)$ sabia que se trata de uma doença não transmissível. Apenas $61 \%$ consideraram o fumo como o principal fator de risco e somente $35,3 \%$ sabiam que a associação de fumo e álcool aumenta consideravelmente o risco de desenvolver a doença. Poucos escolares $(39,2 \%)$ afirmaram ter conhecimento do autoexame de boca. O nível de conhecimento dos escolares sobre os fatores de risco e prevenção do câncer de boca foi baixo. O desconhecimento do autoexame é preocupante, pois esse procedimento pode contribuir para o diagnóstico precoce da doença, aumentando a sobrevida do paciente.

Descritores: Avaliação em Saúde; Conhecimento; Adolescente; Fatores de Risco; Neoplasias Bucais.

\section{Abstract}

The knowledge of the relationship between oral cancer and factors related to lifestyle, such as smoking, drinking and sun exposure are known in the scientific community, however, this knowledge must also be disseminated in the different strata of the population. The objective was to evaluate the knowledge of students of the 3rd year of high school in a medium-sized municipality in the State of São Paulo about oral cancer. A cross-sectional study with 1012 students from public schools in Araçatuba-SP, Brazil was realized. A structured questionnaire containing questions about general knowledge was used; symptomatology; risk factors; protection factors; epidemiology; prevention and treatment of oral cancer. The data were processed and analyzed with the aid of the Epilnfo version 7.2 program. Most students (93.9\%) had heard about oral cancer, however, just over half $(60.1 \%)$ knew that it is a non-communicable disease. Only $61 \%$ considered smoking as the main risk factor and only $35.3 \%$ knew that the association of smoking and alcohol considerably increases the risk of developing the disease. Few students (39.2\%) said they were aware of self-examination by mouth. The level of knowledge of the students about the risk factors and prevention of oral cancer was low. The lack of self-examination is worrying, as this procedure can contribute to the early diagnosis of the disease, increasing the patient's survival.

Descriptors: Health Evaluation; Knowledge; Adolescent; Risk Factors; Mouth Neoplasms.

\section{Resumen}

El conocimiento de la relación entre el cáncer bucal y factores relacionados con el estilo de vida, como el tabaquismo, la bebida y la exposición al sol, son conocidos en la comunidad científica, sin embargo, este conocimiento también debe ser difundido en los diferentes estratos de la población. El objetivo fue evaluar el conocimiento de estudiantes de 3er año de secundaria de un municipio mediano del Estado de São Paulo sobre el cáncer bucal. Estudio transversal con 1012 estudiantes de escuelas públicas de Araçatuba-SP, Brasil. Se utilizó un cuestionario estructurado que contenía preguntas sobre conocimientos generales; sintomatología; factores de riesgo; factores de protección; epidemiología; prevención y tratamiento del cáncer oral. Los datos fueron procesados y analizados con la ayuda del programa Epilnfo versión 7.2. La mayoría de los estudiantes $(93,9 \%)$ había oído hablar del cáncer oral, sin embargo, poco más de la mitad $(60,1 \%)$ sabía que se trata de una enfermedad no transmisible. Solo el $61 \%$ consideraba al tabaquismo como el principal factor de riesgo y solo el $35,3 \%$ sabía que la asociación del tabaquismo y el alcohol aumenta considerablemente el riesgo de desarrollar la enfermedad. Pocos estudiantes $(39,2 \%)$ dijeron que eran conscientes del autoexamen oral. El nivel de conocimiento de los estudiantes sobre los factores de riesgo y prevención del cáncer bucal fue bajo. La falta de autoexamen es preocupante, ya que este procedimiento puede contribuir al diagnóstico precoz de la enfermedad, aumentando la supervivencia del paciente.

Descriptores: Evaluación en Salud; Conocimiento; Adolescente; Factores de Riesgo; Neoplasias de la Boca.

INTRODUÇÃO

As neoplasias malignas representam 0 principal problema de saúde pública no mundo e, na maioria dos países, estão entre as quatro maiores causas de morte antes dos 70 anos de idade. No Brasil, as estimativas sugerem que, em cada ano do triênio 2020-2022, ocorrerão 625 mil casos novos da doença ${ }^{1}$. Dentre os diversos tipos, destaca-se o câncer de boca, uma doença multifatorial, que possui etiologia exógena, envolvendo componentes ambientais, e endógena, relacionada a fatores genéticos ${ }^{2}$.
No ano de 2017, foram registrados, no Brasil, 4.923 óbitos por câncer de boca em homens e 1.372 em mulheres, entretanto, as estimativas para o triênio 2020-2022 são ainda mais preocupantes, pois estima-se que, em cada ano deste período, ocorrerão 11.180 casos da doença em homens e 4.010 em mulheres, correspondendo a um risco de 10,69 casos novos a cada 100 mil homens e de 3,71 para cada 100 mil mulheres, ocupando, respectivamente, a quinta e a décima terceira 
posição entre os tipos de câncer mais frequentes ${ }^{1}$.

O tratamento do câncer de boca está associado à redução da qualidade de vida dos pacientes, devido às mudanças funcionais, estéticas e emocionais significativas, com deterioração de funções básicas, como mastigação, respiração, salivação, deglutição e fala $^{3,4}$. O aumento no risco do aparecimento do câncer de boca devido aos fatores ambientais, principalmente os relacionados ao estilo de vida, como o tabagismo, o etilismo e a exposição solar inadequada são bem conhecidos na comunidade científica, entretanto, o conhecimento sobre a importância desses fatores diminui em diversos estratos da população ${ }^{5-7}$. Ademais, gera preocupação o fato de que o contato dos adolescentes com fatores ambientais e hábitos nocivos à saúde, como o tabagismo e o etilismo tem ocorrido de forma cada vez mais precoce ${ }^{8,9}$.

Neste contexto, torna-se fundamental compreender o nível de conhecimento dos jovens sobre o câncer de boca, visando a identificação das principais deficiências e 0 planejamento adequado de ações de prevenção e educação em saúde direcionados para essa população. Assim, o objetivo deste estudo foi avaliar o conhecimento de escolares do $3^{\circ}$ ano do ensino médio sobre o câncer de boca, com ênfase em seus fatores de risco e prevenção.

MATERIAL E MÉTODO

Trata-se de um estudo observacional, transversal, analítico, do tipo inquérito, realizado com escolares do $3^{\circ}$ ano do ensino médio das escolas da rede pública de ensino de um município de médio porte do Estado de São Paulo. Todos os alunos regularmente matriculados, de ambos os sexos e independente da idade, foram convidados a participar da pesquisa, e aqueles cujos responsáveis legais não assinaram o termo de consentimento livre e esclarecido, aqueles que não concordaram em participar da pesquisa, e aqueles que estavam ausentes após três tentativas de coleta de dados foram excluídos do estudo.

Um questionário estruturado foi elaborado com base em dados da literatura científica, informações disponibilizadas no endereço eletrônico do Instituto Nacional de Câncer José Alencar Gomes da Silva e sugestões de profissionais especialistas na área de oncologia. Os temas abordados foram: conhecimentos gerais; sintomatologia; fatores de risco; fatores de proteção; epidemiologia; prevenção e tratamento do câncer de boca. Previamente à aplicação do questionário, um estudo piloto foi realizado em três escolas não incluídas na amostra da pesquisa para verificar a necessidade de adequações no instrumento de coleta de dados.

A coleta de dados foi realizada por um único pesquisador, por meio de aplicação supervisionada do questionário nas salas de aulas das escolas, assegurando que não haveria troca de informações entre os escolares ou consulta a fontes externas de informações, evitando a ocorrência de vieses nas respostas.

$$
\text { Os dados foram analisados }
$$

empregando-se técnicas de estatística descritiva e os resultados foram apresentados sob a forma de tabelas e gráficos. Foi aplicado o teste de comparação de duas proporções, adotando-se o nível de significância de $5 \%$, e o processamento e análise dos dados foi realizada com auxílio do software Epi Info versão 7.2.

A pesquisa foi aprovada pelo Comitê de Ética em Pesquisa em seres humanos da Faculdade de Odontologia de AraçatubaUNESP (Processo no 1504/09) e foi realizada de acordo com as normas da Declaração de Helsinque e das Resoluções no 466/2012 e 510/2016 do Conselho Nacional de Saúde.

RESULTADOS

No total, 1012 escolares participaram da

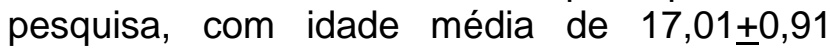
anos. Em relação ao conhecimento sobre a doença, verificou-se que a maioria dos escolares $(93,9 \%)$ já havia ouvido falar sobre o câncer de boca, entretanto, apenas $60,1 \%$ sabia que o câncer se trata de uma doença não transmissível. Observou-se que $30,5 \%$ dos escolares responderam erroneamente que a doença é causada devido à penetração de vírus nas células, enquanto a resposta correta, crescimento desordenado das células, foi respondida por apenas $25,4 \%$.

Sobre a sintomatologia do câncer de boca, aproximadamente $70 \%$ dos escolares responderam que, no início, o câncer não dói, entretanto, somente $38,9 \%$ identificaram a dificuldade de falar; dificuldade de mastigar; dificuldade de engolir e emagrecimento rápido como sintomas de fases mais avançadas da doença.

Em relação aos fatores de risco do câncer de boca, a grande maioria dos escolares sabia que o hábito de fumar pode prejudicar a saúde do indivíduo (98\%), além de prejudicar também a saúde das pessoas que estão próximas ao fumante $(98,3 \%)$. Observou-se que não houve diferença estatisticamente significativa $(p=0,6186)$ entre a proporção de respostas corretas para essas variáveis. 
Embora a grande maioria dos escolares saiba que fumar é prejudicial à saúde, uma proporção significativamente menor $(p<0,0001)$ sabia que não existem doses seguras para 0 uso do cigarro $(88 \%)$ e conhecia a grande quantidade e variedade de substâncias químicas presentes no produto $(55,4 \%)$.

Conforme observado na figura 1,0 hábito de fumar foi considerado como o principal fator de risco para o câncer de boca por aproximadamente $60 \%$ dos escolares, uma proporção significativamente menor $(p<0,0001)$ em comparação àquela que apresentou conhecimento sobre o prejuízo do cigarro sobre a saúde do indivíduo.

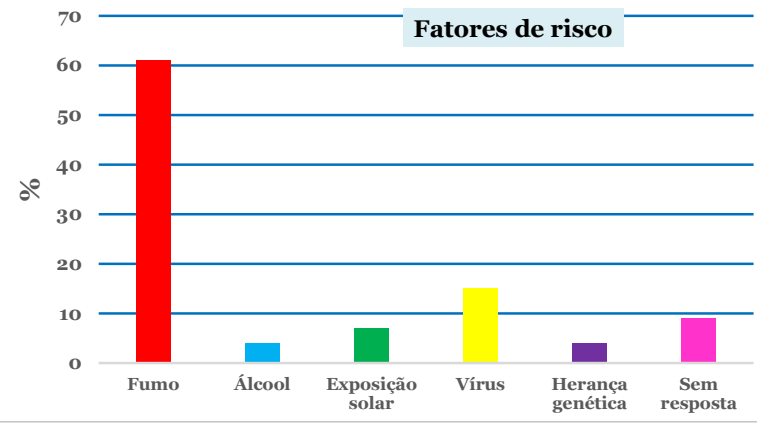

Figura 1: Principal fator de risco para o câncer de boca relatado por alunos do $3^{\circ}$ ano do Ensino Médio.

Similarmente, como demonstrado na figura 2, a associação entre o fumo e o álcool, só foi reconhecida como fator de risco para a doença por pouco mais de um terço dos escolares $(p<0,0001)$.

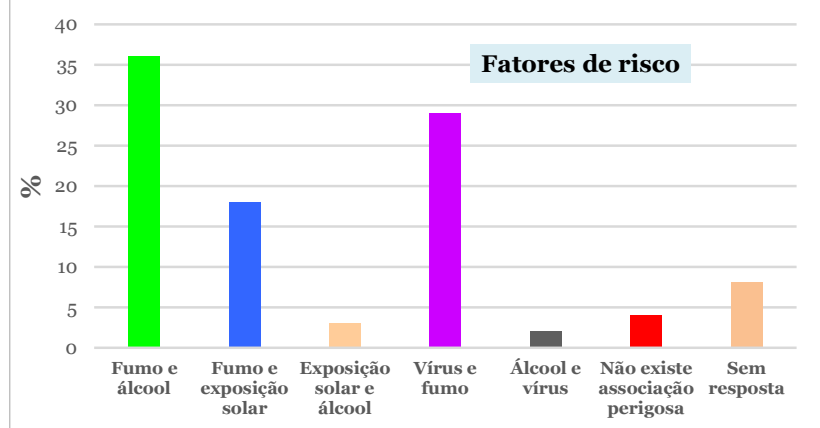

Figura 2: Associação dos fatores de risco para câncer de boca relatados por alunos do $3^{\circ}$ ano do Ensino Médio.

Sobre os fatores de proteção do câncer de boca, verificou-se que $63,4 \%$ dos escolares assinalaram a resposta que agrupava os fatores alimentação saudável; não fumar; não consumir bebida alcoólica e proteger-se contra o sol como medidas preventivas para a doença; e $70,2 \%$ identificaram as frutas e os vegetais como alimentos adequados para tal finalidade.

Em relação à epidemiologia do câncer de boca, observou-se que embora $72,8 \%$ dos escolares tenham afirmado corretamente que a doença ocorre mais em homens do que em mulheres, apenas 20,3\%, uma proporção significativamente menor $(p<0,0001)$, tinha conhecimento sobre as taxas de incidência da doença em homens e mulheres. A grande maioria desconhecia a faixa etária em que o câncer bucal ocorre com maior frequência, sendo que apenas $16,9 \%$ afirmaram corretamente ser entre 40 e 60 anos de idade.

Verificou-se que $86,9 \%$ dos escolares acreditam que o câncer de boca tem cura, entretanto, houve uma diferença significativa em relação à proporção de alunos que conheciam métodos de detecção precoce da doença, pois menos de $40 \% \quad(p<0,0001)$ afirmaram ter conhecimento do autoexame de boca (Figura 3) e $76,9 \%(p<0,0001)$ procuraria o médico ou 0 cirurgião-dentista caso encontrasse uma ferida na boca. Sobre o tratamento da doença, observou-se que somente $31,5 \% \quad(p<0,0001)$ tinham conhecimento que cirurgia, radioterapia e quimioterapia são tratamentos para o câncer de boca, demonstrando que embora acreditam que o câncer de boca tem cura, não conhecem os meios para sua detecção precoce e tratamento.

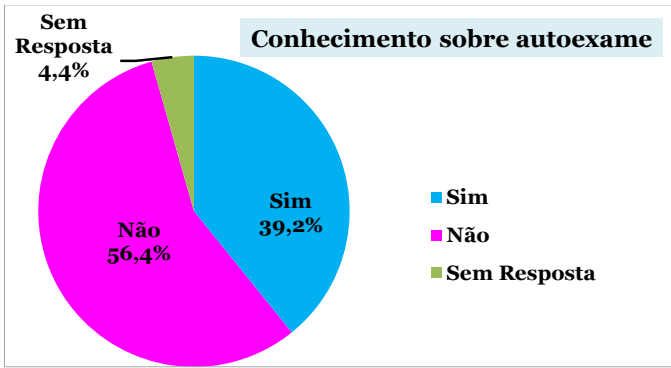

Figura 3: Conhecimento sobre o autoexame de câncer de boca pelos alunos do $3^{\circ}$ ano do ensino médio.

DISCUSSÃO

No presente estudo, o nível de conhecimento sobre o câncer de boca em escolares do $3^{\circ}$ ano do ensino médio mostrouse limitado e apresentou importantes lacunas, especialmente considerando os fatores de risco e métodos de detecção precoce da doença.

Similarmente, outros estudos também verificaram que alunos do ensino médio, bem como parte considerável da população em geral, não possui conhecimento suficiente sobre o câncer bucal ${ }^{10,11}$. Nota-se que o baixo conhecimento sobre a doença pode ser devido à falta de esclarecimentos específicos, principalmente àqueles relacionados aos fatores etiológicos e às situações de risco. Nesse sentido, evidencia-se a necessidade de estratégias de educação em saúde para conscientizar as pessoas sobre os fatores de risco, apresentação clínica inicial e sintomas, a fim de melhorar a prevenção e otimizar as chances de diagnóstico precoce. Ademais, dados da literatura têm demonstrado que o 
conhecimento e a conscientização sobre o câncer de boca precisam ser aprimorados, mesmo entre estudantes de odontologia e medicina, sugerindo que a melhora do currículos educacionais em relação ao estudo da doença e o aumento do contato dos estudantes com pacientes com lesões orais, incluindo o câncer boca, podem ajudar a melhorar sua capacidade diagnóstica futura e práticas de detecção precoce ${ }^{12,13}$

A popularidade contínua do tabagismo parece desafiar uma explicação racional, pois, como observado no presente estudo, os jovens geralmente reconhecem que o fumo causa malefícios à sua própria saúde e para as pessoas ao seu redor, entretanto, o contato cada vez mais precoce dos indivíduos com o cigarro gera preocupação constante. A introdução precoce e o tabagismo persistente durante a adolescência continuam sendo uma ameaça à saúde pública, visto que aproximadamente $80 \%$ dos fumantes crônicos adultos relatam ter começado o hábito de fumar antes dos 18 anos $^{14,15}$

O tabagismo é o principal fator de risco para o surgimento do câncer de boca, e quando associado ao consumo de álcool, a combinação aumenta consideravelmente as chances de desenvolvimento da doença ${ }^{16,17}$. Preocupantemente, somente pouco mais da metade dos escolares desta pesquisa consideraram o cigarro como principal fator de risco para o câncer de boca, revelando uma importante deficiência na relação entre o conhecimento científico existente e a conscientização da população. Outros estudos encontraram resultados semelhantes ou ainda piores aos observados nesta pesquisa e sugerem que estratégias de saúde pública mais elaboradas, incluindo o uso de mídias sociais, jornais, rádio, televisão, e atuação de lideranças e representantes de comunidades podem ser úteis como estratégia para que as informações atinjam, de forma compreensível e adequada, os diferentes estratos populacionais ${ }^{18,19}$.

Embora o mecanismo envolvido nessa alteração não seja totalmente compreendido, sabe-se que os fatores de risco tabagismo e consumo de álcool têm atuação sinérgica para o desenvolvimento do câncer de boca, entretanto, a gravidade dessa associação ainda não está bem esclarecida para os adolescentes, conforme constatado nos achados do presente estudo. De fato, a literatura demonstra que o conhecimento da população sobre a influência do consumo de álcool como fator de risco para o câncer de boca é insuficiente, principalmente entre os indivíduos com menor nível de escolaridade 20,21 . Como esperado, quando comparado com a população em geral, o nível de conhecimento sobre os fatores de risco do câncer de boca é maior entre os cirurgiõesdentistas e estudantes de odontologia e os estudos reforçam a necessidade de aprimorar os currículos odontológicos em relação ao treinamento clínico do exame e detecção precoce da doença ${ }^{22,23}$. Essa discrepância no nível de conhecimento em relação à população pode ser devido ao fato de os profissionais de saúde não dispensarem tempo suficiente ao planejamento e desenvolvimento de atividades preventivas e educativas sobre o câncer de boca junto aos pacientes, tanto em ações coletivas quanto em atendimentos individuais. Nesse sentido, a realização de atividades educativas envolvendo os escolares pode ser de fundamental importância, pois o cenário escolar é propício para o aprendizado e esclarecimento de dúvidas.

O consumo de álcool por adolescentes é um importante problema de saúde pública que pode ter impactos sociais severos, incluindo baixo desempenho acadêmico, vitimização física, suicídio e morte ${ }^{24}$. Estudos indicam que dois em cada três alunos do ensino médio relatam uso de álcool e evidenciam que é necessário melhorar as estratégias de atenção e cuidado aos adolescentes que fazem uso de álcool, além de melhorar a compreensão sobre os fatores que levam ao início precoce do hábito, visando reduzir o comportamento de risco e prevenir consequências negativas ${ }^{24,25}$.

Em contrapartida aos fatores de riscos estão os fatores protetores para o câncer de boca. O tabagismo e o consumo de álcool são conhecidos como os principais fatores de risco para o câncer de boca, entretanto, a dieta também desempenha um papel importante na etiologia da doença. Nesse sentido, a literatura demonstra que 0 alto consumo de vegetais frescos, frutas, peixes e frutos do mar podem contribuir para a prevenção do câncer de boca $^{26}$. Assim, nota-se a importância de conscientizar os jovens sobre a adoção de hábitos alimentares saudáveis, visando não apenas a prevenção do câncer de boca, mas também de problemas relacionados a distúrbios metabólicos, como a obesidade.

Considerando a alta taxa de mortalidade e as sequelas significativas das neoplasias malignas de cavidade oral, diversas campanhas de prevenção do câncer de boca são realizadas, com o intuito de orientar e elucidar a população quanto aos fatores de risco e à importância do diagnóstico de lesões em estágio precoce ${ }^{27}$. Nesse contexto, destaca-se a importância da 
popularização do autoexame de boca, conhecido por apenas $39,2 \%$ dos escolares consultados no presente estudo, por se tratar de um procedimento relativamente simples de ser executado e que não necessita de equipamentos específicos ou materiais de custo financeiro elevado. Apesar de baixa, esta porcentagem ainda foi maior que a observada em estudo realizada em alunos do último ano do ensino fundamental e do primeiro ano do ensino médio de escolas públicas de Santa Catarina, o qual verificou que apenas $27,9 \%$ tinham conhecimento sobre o autoexame da cavidade oral $^{28}$.

A prevenção e o diagnóstico precoce da doença podem ajudar a reduzir a mortalidade do câncer de boca, uma vez que quanto mais cedo o paciente detecta a presença da lesão e procura o tratamento adequado, mais favorável será o prognóstico. Nesse contexto, ações para orientação e conscientização dos escolares são fundamentais, pois podem transformar o aluno de um simples receptor de informações para um propagador de conhecimentos para o interior de seu núcleo social ${ }^{29}$.

CONCLUSÃO

Existe uma importante lacuna no conhecimento sobre a etiologia e fatores de risco do câncer de boca entre os escolares do ensino médio. O desconhecimento do autoexame de boca é preocupante, uma vez que, por meio deste procedimento, o câncer pode ser diagnosticado precocemente, aumentando consideravelmente a sobrevida do paciente.

\section{REFERENCIAS}

1. Instituto Nacional de Câncer José Alencar Gomes da Silva. Estimativa 2020: incidência de câncer no Brasil. Rio de Janeiro: Inca; 2019.

2. Rivera $\mathrm{C}$. Essentials of oral câncer. Int $\mathrm{J}$ Clin Exp Pathol. 2015;8(9):11884-94.

3. Rigoni L, Bruhn RF, de Cicco R, et al. Quality of life impairment in patients with head and neck cancer and their caregivers: a comparative study. Braz J Otorhinolaryngol. 2016;82:680-86.

4. Barder B, Dergousoff J, Nesbitt $M$, et al. Depression as a predictor of postoperative functional performance status (PFPS) and treatment adherence in head and neck cancer patients: a prospective study. J Otolaryngol Head Neck Surg. 2015;44:38.

5. Lee TY, Tseng YH. The potential of phytochemicals in oral cancer prevention and therapy: a review of the evidence. Biomolecules. 2020;10(8):E1150.

6. Nagao T, Warnakulasuriya S. Screening for oral cancer: Future prospects, research and policy development for Asia. Oral Oncol. 2020;105:104632.
7. Osazuwa-Peters N, Adjei Boakye E, Hussaini AS, et al. Characteristics and predictors of oral cancer knowledge in a predominantly African American community. PLoS One. 2017;12(5): e0177787.

8. Ahun MN, Lauzon B, Sylvestre MP, et al. A systematic review of cigarette smoking trajectories in adolescentes. Int $\mathrm{J}$ Drug Policy. 2020;83:102838.

9. Zhao X, Kelly AB, Rowland B, et al. Intention to drink and alcohol use before 18 years among Australian adolescents: An extended Theory of Planned Behavior. Addict Behav. 2020;111: 106545.

10. Keten HS, Isik O, Guvenc N, et al. Evaluation of the level of knowledge of oral cancer among high school students. J Cancer Educ. 2017;32(1):79-84.

11. Azimi S, Ghorbani Z, Tennant M, et al. Population survey of knowledge about oral cancer and related factors in the capital of Iran. J Cancer Educ. 2019;34(1):116-23.

12. Radman M, Glavina A, Sabol I, et al. Knowledge of oral cancer among the fourth and fifth year dental students. Acta Stomatol Croat. 2018;52(4):340-47.

13. Hassona $\mathrm{Y}$, Scully C, Abu Tarboush N, et al. Oral cancer knowledge and diagnostic ability among dental students. J Cancer Educ. 2017;32(3):566-70.

14. CDC. Current cigarette smoking among adults United States, 2005-2014. Morb Mortal Wkly Rep. 2015;64(44):1233-240.

15. West R. Tobacco smoking: Health impact, prevalence, correlates and interventions. Psychol Health. 2017;32(8):1018-36.

16. Kumar M, Nanavati R, Modi TG, et al. Oral cancer: Etiology and risk factors: A review. J Cancer Res Ther. 2016;12(2):458-63.

17. Bezerra NV, Leite KL, de Medeiros MM, et al. Impact of the anatomical location, alcoholism and smoking on the prevalence of advanced oral cancer in Brazil. Med Oral Patol Oral Cir Bucal. 2018;23(3):e295-e301.

18. Bajracharya D, Gupta S, Sapkota M, et al. Oral cancer knowledge and awareness in patients visiting kantipur dental college. J Nepal Health Res Counc. 2018;15(3):247-51.

19. Nabirye RC, Kamulegeya A. Public knowledge about oral cancer in Uganda: a free dental camp experience. J Health Res. 2019;33(4): 270-79.

20. Osazuwa-Peters N, Adjei Boakye E, Hussaini AS, et al. Characteristics and predictors of oral cancer knowledge in a predominantly African American community. PLoS One. 2017;12(5):e0177787.

21. Shimpi N, Jethwani $M$, Bharatkumar $A$, et al. Patient awareness/knowledge towards oral cancer: a cross-sectional survey. BMC Oral Health. 2018;18(1):86. 
22. Kebabcıoğlu Ö, Pekiner FN. Assessing oral cancer awareness among dentists. J Cancer Educ. 2018;33(5):1020-26.

23. Dumitrescu AL, Ibric S, Ibric-Cioranu V. Assessing oral cancer knowledge in Romanian undergraduate dental students. J Cancer Educ. 2014;29(3):506-13.

24. Carpenter RW, Padovano HT, Emery NN, et al. Rate of alcohol consumption in the daily life of adolescents and emerging adults. Psychopharmacology (Berl). 2019;236(11): 3111-124.

25. Borrás Santiesteban T. Alcohol consumption in early adolescence and medical care. Arch Argent Pediatr. 2016;114(5):412-18.

26. Chen F, Yan L, Lin L, et al. Dietary score and the risk of oral cancer: a case-control study in southeast China. Oncotarget. 2017;8(21): 34610-616.

27. Nemoto RP, Victorino AA, Pessoa GB, et al. Campanha de prevenção do câncer de boca: estamos atingindo o verdadeiro público-alvo?. Braz J Otorhinolaryngol. 2015;81(1):44-9.

28. Comunello IF, Bottan ER, Marin $\mathrm{C}$, et al. Assessment of knowledge about oral cancer: study with students of public education. RSBO (Online). 2015;12(1):41-9.

29. Lee $A$, Lo $A$, Li $Q$, et al. Health promoting schools: an update. Appl Health Econ Health Policy. 2020;18(5):605-23.

\section{CONFLITO DE INTERESSES}

Os autores declaram não haver conflitos de interesse

\section{AUTOR PARA CORRESPONDÊNCIA}

\begin{tabular}{l}
\hline Fernando YamamotoChiba \\
Departamento de Odontologia Preventiva e \\
Restauradora, \\
Universidade Estadual Paulista (UNESP) \\
Faculdade de Odontologia de Araçatuba \\
16015-050 Araçatuba - SP, Brasil \\
E.mail: fernando.chiba@unesp.br
\end{tabular}

Submetido em 28/12/2020 Aceito em 16/07/2021 